\title{
Adesão ao checklist cirúrgico para a segurança do paciente: percepção da equipe de enfermagem
}

\author{
Adherence to the surgical checklist for patient safety: perception of the nursing team \\ Cumplimiento de la lista de verificación quirúrgica para la seguridad del paciente: \\ percepción del equipo de enfermería
}

\begin{abstract}
Claudiana Moreira Magalhães ${ }^{1 *}$, Cristiany Feitosa da Silva Neves ${ }^{1}$, Eloíde Neves Coelho, Marcela Milrea Araújo Barros ${ }^{1}$.
\end{abstract}

\begin{abstract}
RESUMO
Objetivo: Descrever a percepção da equipe de enfermagem que atua no centro cirúrgico em relação à adesão do preenchimento do checklist cirúrgico. Métodos: Trata-se de um estudo investigatório-descritivo, sustentado pela abordagem qualitativa, realizado em um hospital público na região Norte do Brasil. A amostra foi composta por 33 profissionais enfermeiros, técnicos de enfermagem e instrumentadores cirúrgicos. Resultados: A partir da análise do conteúdo, os resultados foram agrupados em três categorias: Os significados atribuídos pela equipe de enfermagem quanto ao uso do checklist, dificuldades enfrentadas para a sua implementação e, a importância da qualificação da equipe o uso do checklist cirúrgico. Os relatos registram que o uso do checklist é uma potente estratégia para evitar erros cirúrgicos e reduz danos aos pacientes, porém, são barreiras às falhas de comunicação, resistência da equipe médica e sobrecarga de trabalho. Conclusão: A qualificação em serviço através da educação permanente em saúde envolvendo a equipe multiprofissional é estratégica para sensibilização do uso correto desse instrumento. Faz-se necessário o aprimoramento do trabalho em equipe, visto que a aplicação do checklist para uma cirurgia segura fortalece a comunicação interpessoal, minimiza erros e proporciona um ambiente seguro.
\end{abstract}

Palavras-chave: Checklist cirúrgico, Adesão, Segurança do paciente, Enfermagem.

\begin{abstract}
Objective: To describe the perception of the nursing staff working in the operating room in relation to compliance with filling out the surgical checklist. Methods: This is an investigative-descriptive study, supported by a qualitative approach, carried out in a public hospital in Northern Brazil. The sample consisted of 33 professional nurses, nursing technicians and surgical instrumentators. Results: From the content analysis, the results were grouped into three categories: The meanings attributed by the nursing team regarding the use of the checklist, difficulties faced for its implementation and the importance of the qualification of the team and the use of the surgical checklist. Reports show that the use of the checklist is a powerful strategy to avoid surgical errors and reduce harm to patients, however, they are barriers to communication failures, medical team resistance and work overload. Conclusion: In-service qualification through continuing health education involving the multidisciplinary team is strategic to raise awareness of the correct use of this instrument. It is necessary to improve teamwork, as the application of the checklist for safe surgery strengthens interpersonal communication, minimizes errors and provides a safe environment.
\end{abstract}

Keywords: Surgical checklist, Patient safety, Nursing.

\section{RESUMEN}

Objetivo: Describir la percepción del personal de enfermería que trabaja en el quirófano en relación al cumplimiento del llenado del checklist quirúrgico. Métodos: Se trata de un estudio investigativo-descriptivo, apoyado en un abordaje cualitativo, realizado en un hospital público del norte de Brasil. La muestra estuvo conformada por 33 enfermeros profesionales, técnicos de enfermería e instrumentadores quirúrgicos. Resultados: A partir del análisis de contenido, los resultados se agruparon en tres categorías: Los significados atribuidos por el equipo de enfermería en cuanto al uso del checklist, dificultades enfrentadas para su implementación y la importancia de la calificación del equipo y el uso de la lista de verificación quirúrgica. Los informes muestran que el uso de la lista de verificación es una estrategia poderosa para evitar errores quirúrgicos y reducir el daño a los pacientes, sin embargo, son barreras para fallas de comunicación, resistencia del equipo médico y sobrecarga de trabajo. Conclusión: La calificación en servicio a través de la educación continua en salud que involucre al equipo multidisciplinario es estratégica para dar a conocer el correcto uso de este instrumento. Es necesario mejorar el trabajo en equipo, ya que la aplicación del checklist para una cirugía segura fortalece la comunicación interpersonal, minimiza los errores y brinda un ambiente seguro.

Palabras clave: Lista de verificación quirúrgica, Cumplimiento, Seguridad del paciente, Enfermería.

${ }^{1}$ Faculdade Interamericana de Porto Velho - UNIRON, Porto Velho - RO. *E-mail: claudianapvh01@gmail.com 


\section{INTRODUÇÃO}

A Organização Mundial da Saúde (OMS), (2019), introduziu no ano de 2008 a Aliança Mundial para a Segurança do Paciente com o intuito de promover o desenvolvimento de política para melhorar a segurança do paciente e a qualidade dos serviços de saúde prestados aos Estados-membros, visto que uma cirurgia segura pode salvar vidas e prevenir a resistência antimicrobiana. Com base nesse entendimento, foi desenvolvido o manual "Cirurgia Segura Salva Vidas", ajustado pela Agência Nacional de Vigilância Sanitária (ANVISA) e abrangentemente difundido no Brasil (TOTI ICC, et al., 2020).

Incorporado ao Programa Nacional de Segurança do Paciente (PNSP) existe o protocolo de cirurgia segura, que objetiva determinar um padrão a ser adotado para a diminuição de erros e mortalidade cirúrgica, permitindo uma segurança maior nos procedimentos cirúrgicos mediante a lista de verificação de segurança cirúrgica (BRASIL, 2016).

O checklist cirúrgico trata-se de uma lista de verificação que objetiva a segurança cirúrgica, minimiza a ocorrência de óbitos e complicações que podem ser evitadas. De tal maneira, o mesmo pode ser usado para diferentes tipos de cirurgia e serve de referencial para a organização do serviço, pois o registro de cuidados ao paciente vai desde a checagem dos seus dados à simples conferência, impendindo assim, o início de uma série de ocorrências de erros simultâneos (OLIVEIRA BCS, et al., 2021).

$\mathrm{Na}$ literatura científica há indícios de que a adesão ao checklist cirúrgico tem sido pouco debatido, principalmente na América Latina, porém, a análise do uso dessa ferramenta, possibilita a identificação dos probemas e fatores relacionados a sua utilização efetiva objetivando resultados positivos para a segurança do paciente (RIBEIRO HCTC, et al., 2017).

Pesquisas associadas à segurança do paciente e a atuação da equipe de enfermagem na implementação de estratégias para o melhoramento da segurança e qualidade dos cuidados são necessários e, simultaneamente inovadores e modernos, podendo colaborar com o conhecimento das causas e efeitos à saúde do paciente, além de subsidiar a partir de evidências científicas, à implantação de estratégias de treinamentos oportunos, à prevenção de eventos adversos e prática da cultura da segurança nos serviços de saúde em geral (OLIVEIRA RM, et al., 2016).

Destarte, se faz imprescindível discussão sobre este tema, pois quando a equipe de enfermagem se mostra interessada e envolvida com a segurança do paciente, é capaz de participar de estratégias de prevenção de erros e, consequentemente, potencializar a segurança (PANZETTI TMN, et al., 2020).

Nesse contexto, a pesquisa foi conduzida a partir da seguinte questão norteadora: Qual a percepção da equipe de enfermagem que atuam no Centro Cirúrgico (CC) em relação à adesão ao preenchimento do checklist para cirurgia segura? $\mathrm{O}$ estudo tem por objetivo descrever a percepção da equipe de enfermagem que atua no centro cirúrgico em relação à adesão do preenchimento do checklist cirúrgico.

\section{MÉTODOS}

Trata-se de uma pesquisa de campo com escopo investigatório e descritivo de abordagem qualitativa. $\mathrm{O}$ cenário do estudo foi o CC de um hospital e pronto socorro público no Norte do Brasil. Foram estabelecidos como critérios de inclusão: a equipe de enfermagem (enfermeiros, técnicos de enfermagem e instrumentadores cirúrgicos), sendo homens e mulheres, sem faixa etária definida que atuam no CC do hospital que aceitaram participar voluntariamente do estudo assinando o Termo de Consentimento Livre Esclarecido (TCLE). Foram excluídos os profissionais em período de férias, licença ou afastamento durante o período de coleta de dados.

A amostra foi constituída de forma não probabilística, do tipo intencional ou proposital, a partir de convite com abordagem individual, conforme disponibilidade de tempo do convidado, sem interferência na continuidade do trabalho, obedecendo ao estabelecido nas normas institucionais e uso de equipamentos de proteção individual em consideração a situação de pandemia da COVID-19. Salientamos que previamente a coleta de dados, o participante somente seguiria com a participação do estudo após leitura prévia e 
assinatura do TCLE. O instrumento foi preenchido em espaço reservado, garantindo o sigilo, a privacidade e a sua participaçao foi voluntária. Utilizou-se como instrumento de coleta de dados, um questionário com 06 perguntas abertas, preenchido e recolhido com tempo aproximado de resposta de 20 a 30 minutos.

Os dados foram analisados a partir da análise de conteúdo e comparados com os achados na literatura científica pertinente. Os participantes foram identificados pela letra maiúscula inicial da categoria profissional: Enfermeiro (E), Técnico de enfermagem (T) e Instrumentador cirúrgico (IC) seguido pela ordem numérica de preenchimento do instrumento.

O estudo foi desenvolvido obedecendo ao estabelecido na Resolução n. ${ }^{\circ}$ 466/2012 do Conselho Nacional de Saúde/Ministério da Saúde. Por se tratar de pesquisa com seres humanos, o estudo foi submetido ao Comitê de Ética em Pesquisa (CEP) da UNINORTE e aprovado com número CAAE: 42544920.3.0000.8028 e Parecer Consubstanciado n.. 4.580 .770 de 09 de março de 2021.

\section{RESULTADOS}

A amostra final foi composta por 33 profissionais de enfermagem (enfermeiros, técnicos de enfermagem e instrumentadores cirúrgicos) com predominância no sexo feminino. O Quadro 1 registra a caracterização dos participantes do estudo.

Quadro 1 - Caracterização dos participantes do estudo, 2021.

\begin{tabular}{|c|c|c|c|}
\hline EQUIPE & SEXO & FAIXA ETÁRIA & TEMPO DE ATUAÇÃO \\
\hline E1 & $\mathrm{F}$ & 20 a 30 anos & 1 a 10 anos \\
\hline E2 & $\mathrm{F}$ & 31 a 40 anos & 1 a 10 anos \\
\hline E3 & $\mathrm{F}$ & 31 a 40 anos & 1 a 10 anos \\
\hline E4 & $M$ & Acima dos 50 anos & 11 a 20 anos \\
\hline E5 & $\mathrm{F}$ & Acima dos 50 anos & 11 a 20 anos \\
\hline E6 & $\mathrm{M}$ & 31 a 40 anos & 1 a 10 anos \\
\hline E7 & $\mathrm{F}$ & 20 a 30 anos & 1 a 10 anos \\
\hline E8 & $\mathrm{F}$ & 41 a 50 anos & 11 a 20 anos \\
\hline E9 & $\mathrm{F}$ & 31 a 40 anos & 1 a 10 anos \\
\hline E10 & $\mathrm{F}$ & 31 a 40 anos & 1 a 10 anos \\
\hline E11 & $\mathrm{F}$ & 31 a 40 anos & 1 a 10 anos \\
\hline $\mathrm{T} 1$ & $\mathrm{~F}$ & 20 a 30 anos & 11 a 20 anos \\
\hline T2 & $\bar{M}$ & 41 a 50 anos & 1 a 10 anos \\
\hline T3 & $\mathrm{F}$ & 20 a 30 anos & 1 a 10 anos \\
\hline T4 & M & 41 a 50 anos & 11 a 20 anos \\
\hline T5 & $\mathrm{F}$ & 31 a 40 anos & 1 a 10 anos \\
\hline T6 & $\mathrm{F}$ & 41 a 50 anos & 11 a 20 anos \\
\hline $\mathrm{T7}$ & $\mathrm{M}$ & 31 a 40 anos & 1 a 10 anos \\
\hline T8 & $\mathrm{F}$ & 31 a 40 anos & 1 a 10 anos \\
\hline T9 & $\mathrm{F}$ & 20 a 30 anos & $1 \mathrm{a} 10$ anos \\
\hline T10 & $\mathrm{F}$ & 41 a 50 anos & 11 a 20 anos \\
\hline T11 & $\mathrm{M}$ & 41 a 50 anos & 1 a 10 anos \\
\hline T12 & $\mathrm{F}$ & Acima dos 50 anos & 11 a 20 anos \\
\hline IC1 & $\mathrm{F}$ & 20 a 30 anos & 1 a 10 anos \\
\hline IC2 & $\mathrm{F}$ & 20 a 30 anos & 1 a 10 anos \\
\hline IC3 & $\mathrm{F}$ & 31 a 40 anos & 11 a 20 anos \\
\hline IC4 & $\mathrm{F}$ & 41 a 50 anos & 1 a 10 anos \\
\hline IC5 & $\mathrm{F}$ & 20 a 30 anos & 1 a 10 anos \\
\hline IC6 & $\mathrm{F}$ & 41 a 50 anos & 11 a 20 anos \\
\hline IC7 & $\mathrm{F}$ & Acima dos 50 anos & 11 a 20 anos \\
\hline IC8 & $\mathrm{F}$ & 31 a 40 anos & 1 a 10 anos \\
\hline IC9 & $\mathrm{F}$ & 20 a 30 anos & 1 a 10 anos \\
\hline IC10 & $\mathrm{F}$ & 41 a 50 anos & 11 a 20 anos \\
\hline
\end{tabular}

Fonte: Magalhães CM, et al., 2021. 
Quanto à equipe de enfermagem, houve um predomínio de profissionais da categoria técnico de enfermagem, isto é justificado, pois de acordo com a Resolução $N^{\circ} 543 / 2017$ do Conselho Federal de Enfermagem, para um cuidado intermediário a equipe deve ser composta por $33 \%$ de enfermeiros e os demais auxiliares e/ou técnicos de enfermagem (CONSELHO FEDERAL DE ENFERMAGEM (COFEN), 2017).

Em pesquisa realizada por Correia MITD, et al. (2019), em relação à idade dos profissionais, houve uma variação de 20 a 50 anos ou mais, perfazendo uma média de 32 anos, corroborando com nosso estudo e evidenciando a prevalência de uma força de trabalho jovem, com toda a capacidade produtiva. Constatouse também um predomínio de participantes do sexo feminino, o que reflete o padrão encontrado na aréa da enfermagem, compatível com o resultado obtido por Lopes TMR, et al. (2019), que demonstrou que a área da saúde tem tendência à feminização. O sexo feminino predomina na profissão enfermagem, tendo uma relação histórica entre o predomínio e o cuidado, uma atividade referencial da profissão.

A partir da análise dos dados os resultados foram agrupados em categorias temáticas: Os significados atribuídos pela equipe de enfermagem quanto ao uso do checklist; Dificuldades enfrentadas para a implementação do checklist para cirurgia segura e; A importância da qualificação da equipe no uso do checklist cirúrgico.

\section{Significados atribuídos pela equipe de enfermagem quanto ao uso do checklist}

A literatura é pródiga em sinalizar que as medidas de segurança empregues equivalem baixa morbimortalidade em centros cirúrgicos, por isso os pesquisadores apresentam algumas atitudes simples, como checar dados do paciente, informações clínicas do indivíduo e órgãos a serem operados, predispor de equipamentos e materiais com funcionamento adequado, assegurando o sucesso das ferramentas (SANTOS JS, et al., 2017).

A cirurgia segura está sendo cada vez mais discutida nos serviços de saúde. No que concerne os significados atribuídos pela equipe de enfermagem quanto ao seu uso, os participantes descrevem que o checklist deve ser atribuição de todos os integrantes da equipe para garantir a segurança do paciente:

"Serve para a segurança da equipe e do paciente" (T2).

"Para segurança do paciente e dos profissionais de saúde" (E1).

“Totalmente importante, para toda a equipe de enfermagem" (IC2).

"Garantir segurança e qualidade de assistência ao paciente da entrada ao Centro Cirúrgico até à saída do mesmo" (T6).

Silva PHA, et al. (2020), corroboram afirmando em seu estudo que a segurança do paciente está intimamente associada à figura da enfermagem, pois, este profissional tem participação direta na implantação de estratégias de cuidado, por meio de uma assistência humanizada e de qualidade, para tal, o checklist é essencial para o processo.

Para essa implementação, é necessário à incorporação de ferramentas assim como aspectos de humanização adotados pela enfermagem e pela equipe de saúde, de forma a adotar procedimentos que assegurem ao paciente a redução de danos e riscos antes, durante e após a cirurgia (CORONA ARP e PENICHE ACG, 2015).

Oliveira MCB, et al. (2018), descrevem que essa lista confere segurança ao paciente e a sua adesão vem acontecendo nos serviços de saúde do Brasil, embora com algumas dificuldades como a falta de esclarecimentos da equipe sobre a importância da implantação para práticas mais seguras e a sua baixa adesão.

Logo, os relatos permitem identificar que o uso do checklist constitui-se em potente estratégia para evitar riscos e erros na hora da cirurgia e redução de danos aos pacientes na realização de procedimentos cirúrgicos conforme foi demonstrado nas falas abaixo: 
"Importante, pois evita que ocorra acidentes ou erros durante um procedimento cirúrgico" (T8).

"É importante, pois através dele podemos evitar riscos e erros na hora da cirurgia" (IC3).

"Acho muito importante, pois assim o checklist auxilia na redução de danos aos pacientes" (T9).

"É importante para a devida identificação correta do paciente, se o paciente tem ciência do procedimento que irá ser realizado, para a confirmação do procedimento a ser realizado, se o paciente apresenta alergia a algum tipo de medicação e se está de jejum. Para evitar intercorrências operatórias" (E6).

Para a equipe de enfermagem do CC é de suma importância que todos os profissionais tenham conhecimento de todas as etapas do checklist, checando o conhecimento de todos os profissionais envolvidos sobre o procedimento a ser realizado, condições clínicas do paciente, necessidade de algum cuidado especial, preparo adequado para cada procedimento, uso de antibiótico/ tempo de uso (CORONA ARP e PENICHE ACG, 2015).

Em estudo realizado por Elias ACGP, et al. (2015) é descrito que, para prevenir essas possíveis falhas, o CC deve adotar rotinas nas quais é enfatizado que qualquer membro da equipe cirúrgica ou de enfermagem, ao buscar o paciente na sala de preparo cirúrgico, deve confirmar o nome completo do paciente, conferir com o prontuário e pulseira de identificação, e reforçar a orientação ao paciente sobre a pulseira que sinaliza a presença de risco de alergia. A utilização do checklist pode contribuir na redução de danos e desfechos fatais, apesar dos desafios que se revelam diante da frequente falta de preenchimento dos dados (RIBEIRO L, 2019).

\section{Dificuldades enfrentadas para a implementação do checklist cirurgia segura}

Falhas na comunicação entre a equipe de enfermagem e equipe médica podem representar em média $39 \%$ das principais dificuldades para a implementação do checklist para uma cirurgia segura. A prática da equipe sobre a segurança do paciente traz a comunicação como ferramenta essencial para a cultura de segurança nos estabelecimentos assistenciais de saúde (SILVA PHA, et al., 2020). Nesta categoria foram elencadas as principais dificuldades enfrentadas para a implementação do checklist para a cirurgia segura, dentre elas os participantes ratificaram que a falta de comunicação entre a equipe é uma das dificuldades, conforme as narrativas abaixo:

\section{"A falta de comunicação [...]" (E9). \\ "A falta de comunicação da equipe" (IC9).}

O processo de comunicação é fator primordial para garantir que as atividades ocorram de maneira correta e eficaz dentro do CC, devendo acontecer constantemente com o intuito de proporcionar informação e compreensão necessárias à condução das tarefas, e acima de tudo, motivação, cooperação e qualidade da assistência prestada (SANTOS JS, et al., 2017).

No estudo de Grabois V e Oliveira J (2019), a falta de comunicação da equipe e a resistência de alguns profissionais no preenchimento do checklist, estão relacionadas à percepção do profissional em acreditar que este é um papel a mais a ser preenchido. Beccaria LM, et al. (2019), corroboram ao frisarem que a dificuldade encontrada na implementação do checklist baseia-se na falta de comunicação da equipe, na resistência dos profissionais em colaborar no preenchimento do checklist, aumentando assim, a probabilidade da ocorrência de eventos adversos.

Santos JS, et al. (2017), descrevem que muitos erros gerados por falhas no processo de comunicação podem ser irreversíveis e, o uso do checklist objetiva reduzir o atrito causado por situações inesperadas. 0 que diz a respeito ao reconhecimento dos erros, informá-los é o primeiro passo para reduzir falhas. 
Cabe a equipe de enfermagem do CC encontrar estratégias que consigam superar tais dificuldades e que contribuam para que cada vez mais a comunicação no ambiente cirúrgico não seja uma barreira e sim uma ferramenta de segurança do paciente (FERREIRA NCS, et al., 2019). Outra dificuldade narrada pela equipe de enfermagem está relacionada a resistência da equipe médica para a implementação do chekclist cirúrgico:

\section{"[...]acredito que vai ter a resistência dos profissionais" (E9).}

"Resistência da equipe médica (cirurgiões e anestesistas)" (T12).

A resistência da equipe médica presente nos resultados pode ser associada como um fator que leva à baixa adesão da implementação do checklist cirúrgico. É fundamental que as instituições hospitares atentem-se pela necessidade de criar estratégias para um compromentimento maior dos anestesistas e cirurgiões pela segurança do paciente (SOUZA, ATG, et al., 2020).

Para Reyes PC, et al. (2019) esse resultado pode torna-se concernente ao desconhecimento do médico sobre os protocolos estabelecidos e/ou checklists de segurança do paciente. Ademais, muitos cirurgiões e anestesiologistas executam eventualmente no setor conforme suas especialidades, com pouca ou nenhuma participação nas discussões organizacionais sobre segurança do paciente.

De acordo com Grabois V e Oliveira J (2019), a resistência da equipe médica, na percepção destes profissionais, está na burocracia do preenchimento do instrumento, já que para eles os eventos adversos são raros e, então, julgam ser desnecessária a utilização desta tecnologia. Essa resistência pelos integrantes das equipes cirúrgicas (cirurgiões e anestesiologista) para o cumprimento da lista de verificação, também foi constatado no estudo de Panzetti TMN, et al. (2020). No estudo, constatou-se que, entre a equipe, os cirurgiões demonstraram maior resistência em aderir à realização do Protocolo.

Outra dificuldade relatada como fator dificultador para a implementação do checklist cirúrgico está relacionada a sobrecarga de trabalho da equipe de enfermagem:

"Sobrecarga de trabalho na equipe de enfermagem" (IC6).

Os achados se assemelham aos resultados de outros estudos, no qual a sobrecarga de trabalho na equipe de enfermagem é um fator dificultador para o uso do instrumento. A sobrecarga de trabalho indica que são necessárias estratégias para facilitar a adaptação e aumentar a adesão das equipes sobre a implementação do protocolo (CORREIA MITD, et al., 2019).

A literatura aponta inclusive, que a implantação desta ferramenta pode na verdade, contribuir para a redução da sobrecarga de trabalho na equipe, colabora com a segurança do paciente, minimiza riscos a partir da organização das atividades e maior coesão entre as equipes, proporcionando assim, maior qualidade no atendimento (ALPENDRE FT, et al., 2017).

\section{A importância da qualificação da equipe no uso do checklist cirúrgico}

As análises das narrativas evidenciaram a necessidade e a importância da qualificação da equipe multiprofissional para o uso correto do checklist cirúrgico, pois proporciona um entendimento desse instrumento garantindo uma visibilidade da equipe para atingir melhores resultado no processo cirúrgico (SOUZA ATG, et al., 2020). A qualificação reforça a importância e os benefícios da aplicação do instrumento, incentivam seu uso e contribui para a mudança da cultura de segurança da instituição (ARAÚJO MPS e OLIVEIRA AC, 2015). Verifica-se na perspectiva dos participantes que é possível qualificarem o cuidado prestado e trazer benefícios para o paciente:

"Tem que ser feito por profissional qualificado, pois no mesmo tem perguntas de níveis complexo do procedimento é de extrema importância identificá-los" (E8).

"Sim. A qualificação é importante para se ter noção do porque está sendo realizado o checklist, pois sem ele pode acontecer alguma consequência ou dano ao paciente, sendo que o intuito é de tratá-lo" (IC9). 
Para o uso do checklist da cirurgia segura ocorrer de forma adequadamente, a equipe de enfermagem e médica precisam de qualificação, reforçando a importância e as vantagens da aplicação dessa ferramenta, e promovendo sua utilização colaborando para a modificação da cultura de segurança das instituições hospitalares (REYES PC, et al., 2019).

A estratégia somente será potencializada com a participação de toda a equipe cirúrgica, assim a qualificação oferece modificações na rotina, no desempenho da equipe de enfermagem, de cada indivíduo coletivamente e individual e na comunicabilidade entre pessoas (ANDRADE LEL, et al., 2018). Assim, Silva FAA e Silva AGN (2017), afirmam que a realização de ações de qualificação e educação permanente em saúde de maneira participativa colabora para o esclarecimento de dúvidas, exposição de dificuldades e o repensar de estratégias para a implementação do instrumento.

Conforme os relatos podemos afirmar que o checklist cirúrgico é de extrema importância e primordial para o processo cirúrgico e os participantes acham importante haver treinamento sobre esse formulário e a qualificação no checklist oferece a oportunidade a equipe de enfermagem de expor suas preocupações diante da checagem dos itens no processo cirúrgico (SOUZA ATG, et al., 2020). Os achados mostram que a qualificação da equipe na utilização correta dessa ferramenta pode atingir melhores resultados, assegurando uma boa visibilidade ao procedimento cirúrgico (RIBEIRO L, 2019).

Sabe-se que a educação permanente em saúde promove o desenvolvimento holístico do profissional, aproveitando os acontecimentos do ambiente de trabalho em que ele está inserido para melhorar e aperfeiçoar os seus conhecimentos (OLIVEIRA BCS, et al., 2021). É possível perceber que as rodas de conversa são uma potente e versátil ferramenta, podendo ser usada como estratégia na adesão do uso do checklist, possibilitando discutir, refletir e ( $\mathrm{re}$ ) construir concepções e práticas com a equipe de enfermagem. Martins FL, et al. (2019), afirmam que as rodas de conversa produzem conhecimentos coletivos e contextualizados já que são construídas pela fala crítica e escuta sensível de forma lúdica favorecendo o entrosamento e a confiança entre os profissionais de saúde.

Os resultados desta categoria evidenciam a importância da qualificação da equipe de enfermagem para o uso do checklist cirúrgico, nesse contexto o conhecimento da equipe do CC sobre a implantação do checklist gera mais segurança ao paciente.

\section{CONCLUSÃO}

Portanto, é necessário fortalecer o trabalho em equipe, pois a aplicação do checklist cirúrgico visa a comunicação interpessoal, reduzir erros e promover a segurança do paciente cirúrgico, propiciando um ambiente eficiente e seguro. Os resultados mostram que os participantes demonstraram ter conhecimentos cientificamente embasados sobre questões que envolvem a segurança cirúrgica na adesão do preenchimento do checklist cirúrgico para cirurgia segura. Deste modo, estabelece à importância da realização de novos estudos voltados para abordagens que relacionem sobre a adesão do checklist cirúrgico para a prática profissional da equipe de enfermagem a fim de contribuir com uma assistência humanizada, protetiva e eficaz.

\section{REFERÊNCIAS}

1. ARAÚJO MPS, OLIVEIRA AC. A assistência cirúrgica após implantação dos núcleos de segurança do paciente. Revista Enferm Centro Oeste Min, 2015; 1(3): 12-16.

2. ANDRADE LEL, et al. Cultura de segurança do paciente em três hospitais brasileiros com diferentes tipos de gestão. Ciência saúde coletiva, 2018; 1(1): 26-25.

3. ALPENDRE FT, et al. Cirurgia segura: validação de checklist pré e pós-operatório. Revista Latino Americana de Enfermagem, 2017; 1(2): 20-28.

4. BECCARIA LM, et al. Eventos adversos na assistência de enferm. em uma unidade de terapia intensiva. Rev. Bras. Ter. intensiva, 2019; 3(21); 276-282.

5. BRASIL. Ministério da Saúde. Documento de referência para o Programa Nacional de Segurança do Paciente/Ministério da Saúde. Agência Nacional de Vigilância Sanitária. 2016. Disponível em:https://www20.anvisa.gov.br/segurancadopaciente/index.php/publicacoes/item/documento-de-referencia-para-oprograma-nacional-de-seguranca-do-paciente. Acessado em: 19 de junho de 2021. 
6. CORREIA MITD, et al. Segurança e qualidade em cirurgia: a percepção de cirurgiões no Brasil, Rev. Col. Bras. Cir, 2019; 1(2).

7. CORONA ARP, PENICHE ACG. A cultura de segurança do paciente na adesão ao protocolo de cirurgia segura. Rev Sobecc, 2015; 1(2).

8. CONSELHO FEDERAL DE ENFERMAGEM (COFEN). Resolução COFEN 543/2017: 2017. Disponível em: http://www.cofen.gov.br/resolucao-cofen-5432017_51440.html. Acessado em: 20 de maio de 2021.

9. ELIAS ACGP, et al. Avaliação da adesão ao checklist de cirurgia segura em hospital universitário público. Revista Sobecc, 2015; 1(4).

10. FERREIRA NCS, et al. Checklist de Cirurgia Segura: conhecimento e utilização do instrumento na perspectiva dos técnicos de enfermagem. Rev. enferm. Centro-Oeste Min, 2019; 1(2): 142-149.

11. GRABOIS V, OLIVEIRA J. Cirurgia Segura: segurança do paciente. Revista Brasileira Enfermagem, $2019 ; 1$ (3): 1220.

12. LOPES TMR, et al. Ações de enfermagem na segurança do paciente em centro cirúrgico: revisão integrativa da literatura. Coleção de Periódicos Eletrônicos Saúde, 2019; 1(3): 124-130.

13. MARTINS FL, et al. Uma expressão nova para estratégia de educação permanente: rodas de conversa. Saúde em Foco, 2019; 1(2): 124-132.

14. ORGANIZAÇÂO MUNDIAL DA SAÚDE. (OMS). Segundo desafio global para a segurança do paciente: Cirurgias seguras salvam vidas. 2019. Disponível em: https://www.who.int/eportuguese/publications/pt/. Acessado em: 03 de maio de 2021.

15. OLIVEIRA RM, et al. Estratégias para promover segurança do paciente: da identificação dos riscos às práticas baseadas em evidências. Esc Anna Nery, 2016; 18(1): 122-129.

16. OLIVEIRA MCB, et. al. Adesão do cheklist cirúrgico à luz da cultura de segurança do paciente. Rev. Sobecc, 2018; 1(1): 136-140.

17. OLIVEIRA BCS, et al. Segurança do paciente em centro cirúrgico: desafios para a prática de enfermagem. Revista Eletrônica Acervo Enfermagem, 2021; 10(2): 147-152.

18. PANZETTI TMN, et al. Adesão da equipe de enfermagem ao protocolo de cirurgia segura. Revista Eletrônica Acervo Saúde, 2020; 12(2): 235-242.

19. REYES PC, et al. Checklist no Centro Cirúrgico: lista de verificação, Ciência Coletiva Saúde, 2019; 1(2): $12-20$.

20. RIBEIRO HCTC, et al. Adesão ao preenchimento do checklist de segurança cirúrgica. Cad. Saúde Pública 2017; 33(10): 250-260.

21. RIBEIRO L, et al. Checklist de cirurgia segura: adesão ao preenchimento, inconsistências e desafios. Rev Col Bras Cir, 2019; 1(1).

22. SANTOS JS, et al. Teste Piloto de Checklist de Cirurgia Segura: Relato de Experiência. Rev. Enferm UFPI, 2017; 6(1): 76-79.

23. SILVA PHA, et al. Cirurgia segura: análise da adesão dos médicos aos protocolos e seu potencial impacto na segurança do paciente. Rev Col Bras Cir, 2020; 6(47).

24. SILVA FAA, SILVA AGN. Equipe de Enfermagem em Cirurgia Segura: Desafios para adesão ao Protocolo. Rev. Enferm UFPI, 2017; 6(2): 23-29.

25. SOUZA ATG, et al. Segurança do paciente em centro cirúrgico: percepção dos profissionais de enfermagem. Rev. Sobecc, 2020; 2(4).

26. TOTI ICC, et al. Percepções dos profissionais de enfermagem na aplicação do checkist de cirurgia segura. J. nurs. Health, 2020; 2(4): 10-14. 